\title{
Interacción de la auditoría de gestión en el direccionamiento estratégico de cooperativas de transporte.
}

\section{Interaction of the management audit in the strategic direction of transport cooperatives.}

Monica Patricia Mayorga Diaz. 1, Edith Andrea Hervas Paredes. ${ }^{2}$, María Alexandra López Paredes. ${ }^{3}$ Silvia Elizabeth Toaza Tipantasig. ${ }^{4}$

DOI: https://doi.org/10.33262/visionariodigital.v4i2.1214

\begin{abstract}
.
The modern business reality of the different organizations at the global level regarding the evaluation of the fulfillment of objectives, leads to the diagnosis of the interaction of the management audit in the strategic direction, for which it is necessary to consider the inclusion of the components of the social responsibility in its management, is when the urgent need arises for the reports of the execution of this type of audit to form part of the strategic vision of any organization, therefore in the case of passenger transport cooperatives in Ecuador it is necessary assess the influence of the measurement of the social component in the strategic direction, an issue that given its fragmentation in the bibliography, it was determined as a general objective of this work: to structure a research design that allows addressing this situation at its different levels, in this sense for this study a procedure of diagnosis, in which four (4) stages of work characterized in three (3) levels were determined: the first consisting of the cooperative government and the representatives of the financial

\footnotetext{
${ }^{1}$ Universidad Regional Autónoma de Los Andes, Ambato Ecuador, monikmayorgad@gmail.com, ORCID: 00000003-3071-236X

${ }^{2}$ Profesional Independiente, Ambato Ecuador, andrea.95hervas@gmail.com

${ }^{3}$ Universidad Técnica de Ambato, Ambato Ecuador, ma.lopez@uta.edu.ec

${ }^{4}$ GAD Pelileo, Ambato Ecuador, sett1802@gmail.com
} 
area of the cooperative who will be called managers; The second is made up of the external auditors who will be the controllers, and the community representatives considered users that correspond to the third level of the proposed procedure.

Keywords: Social responsibility, cooperative government, external auditors, controllers

\section{Resumen.}

La realidad empresarial moderna de las distintas organizaciones a nivel global en cuanto a la evaluación del cumplimiento de objetivos, conlleva al diagnóstico de la interacción de la auditoría de gestión en el direccionamiento estratégico, por lo que es preciso considerar la inclusión de los componentes de la responsabilidad social en su gestión, es cuando surge la urgente necesidad de que las memorias de la ejecución de este tipo de auditoría formen parte de la visión estratégica de cualquier organización, por consiguiente para el caso de cooperativas de transporte de pasajeros en el Ecuador es menester valorar la influencia de la medición del componente social en el direccionamiento estratégico, tema que dada su fragmentación en la bibliografía, se determinó como objetivo general de este trabajo: estructurar un diseño de investigación que permita abordar esta situación en sus diferentes niveles, en tal sentido para este estudio se diseña un procedimiento de diagnóstico, en el que se determinaron cuatro (4) etapas de trabajo caracterizadas en tres (3) niveles: el primero integrado por el gobierno cooperativo y los representantes del área financiera de la cooperativa que se denominarán gestores; el segundo lo conforman los auditores externos que serán los controladores, y los representantes de la comunidad considerados usuarios que corresponde el tercer nivel del procedimiento planteado.

Palabras claves: Responsabilidad social, gobierno cooperativo, auditores externos, controladores

\section{Introducción.}

En la edad media la auditoría trataba de descubrir fraudes, este enfoque se mantuvo hasta finales del siglo XIX a partir de la revolución industrial la economía se desarrollaba en base a una estructura de empresa familiar, con el pasar el tiempo se creó las grandes sociedades y la admiración quedo dividida y surgió la necesidad de conseguir una adecuada protección, a través de una auditoría independiente que consistía en contratar personas externas a las empresas que de este modo sean imparciales al momento de realizar su trabajo, para que elaboren mecanismos de supervención y control a la actividades que realizaban los trabajadores, posterior mente las relaciones comerciales empezaron a creer rápidamente, lo cual tuvo la necesidad de crear un nuevo sistema de supervisión, de activos y por ende el descubrimiento y prevención de fraudes, el auditor era considerado como un revisor de cuentas, con el avance del tiempo la auditoria se extendió a la 
revisión de la eficiencia de los empleados, procedimientos administrativos, cumpliendo de metas y objetivos. (Sandoval, 2012).

Por el año 1957 surgió la auditoría administrativa con la finalidad de evaluar la calidad de la administración, esto es la planificación, la organización, la dirección y el control de las entidades. Durante la década del 70, apareció el interés por parte de las administraciones gubernamentales de conocer acerca del desempeño de sus entidades, ya que a través de los informes financieros esto ni era viable y nació la auditoría de gestión, basada en dos importantes principios de la administración pública: el primero relativo a que esta debía ser dirigida de manera que hiciera el mejor uso posible de los fondos públicos y el segundo relativo a que las personas que dirigía los fondos públicos debían ser responsables por las prudente y efectiva administración de los recursos que les habían sido confiables. Con posterioridad, dado el grado de socialización de la propiedad bajo asociaciones de diferentes matices y características, lo cual provocó que la administración de los negocios cada vez más recayera en manos de directivos gerenciales que raras veces era a su vez los propietarios principales de las mismas, nació la necesidad de conocer, con un carácter totalmente independiente, acerca del desempeño de las administraciones bajo la influencia de las complejas relaciones y el entorno en que se movían las entidades del sector privado y se reconoció la utilidad de la auditoría de gestión. (De Armas, 2008, p.6)

A partir de algunos referentes bibliográficos se realiza el análisis categorial de la conceptualización y aportes sobre la auditoría de gestión. (tabla 1.)

Tabla 1. Análisis categorial de la conceptualización de auditoría de gestión y su finalidad

\begin{tabular}{|c|c|}
\hline Autor & Categoría de análisis \\
\hline $\begin{array}{l}\text { Sánchez, } 1987 \\
\end{array}$ & $\begin{array}{l}\text { Se aborda el concepto de auditoría operacional conjuntamente Metodología y evaluación } \\
\text { con su metodología, evolución de sistemas, informales y el } \\
\text { seguimiento de sugerencias. }\end{array}$ \\
\hline $\begin{array}{l}\text { Redondo, Llopart, } \\
\text { Duran, } 1996\end{array}$ & $\begin{array}{l}\text { Está asociada a la revisión de los indicadores vinculados con la Indicadores vinculados con la } \\
\text { actuación y posicionamiento desde el punto de vistaactuación y posicionamiento } \\
\text { competitivo }\end{array}$ \\
\hline $\begin{array}{l}\text { Alvarado, Corvera } \\
\text { Márquez }\end{array}$ & $\begin{array}{l}\text { Basados en los principios de la administración pública, a partir Administración pública } \\
\text { de grado de socialización de la propiedad bajo asociaciones. }\end{array}$ \\
\hline Rivas, 2002 & $\begin{array}{l}\text { Se revisan las dinámicas establecidas en las entidades del sector Sector privado } \\
\text { privado. }\end{array}$ \\
\hline Maldonado, 2006 & $\begin{array}{l}\text { Encargada de medir y cuantificar los logros alcanzados por la Medir y cuantificar los logros } \\
\text { empresa en un período determinado de tiempo. }\end{array}$ \\
\hline
\end{tabular}


Fonseca, 2007 Realiza una propuesta de indicadores financieros y de gestión Indicadores financieros y de para establecer desviaciones y sugerir la implementación de gestión.

medidas correctivas y necesarias que generan información cuantitativa y cualitativa del proceso administrativo.

Fernández, 2008

Los Auditores deberán efectuar exámenes de auditoria de forma Exámenes de auditoria objetiva y sistemática y profesional.

Armas R, 2008

Se determinan los hallazgos de auditoría, los mismos que Hallazgos de auditoría contarán con un juicio de valor independiente dentro de un informe de auditoría, el que contiene una matriz de recomendaciones para su seguimiento y monitoreo.

Blanco, 2004

Proponer características uniformes para los documentos deDocumentos de trabajo trabajo dentro de la Auditoría de Gestión.

Estupiñán, 2006

Deficientes existentes deberían contener hallazgos positivos, Hallazgos, incluyendo causas y incluyendo causas y condiciones de cumplimiento, de eficacia condiciones de cumplimiento. y economía de gestión y uso de recursos de la entidad auditada.

Armas, 2008

La auditoría de gestión es la revisión sistemática de las Revisión sistemática de las actividades de una organización o de un segmento de ella en actividades de una relación con objetivos específicos. Tal revisión tiene tres organización propósitos esenciales: estimar el funcionamiento, identificar oportunidades de perfeccionamiento y desarrollar recomendaciones de mejoras o fomentar acciones

Maldonado, 2011 Una auditoría operativa es un examen objetivo y sistemático Examen objetivo y sistemático de evidencias con el fin de proporcionar una evoluciónde evidencias. independiente del desempeño de una organización, programa, actividad o función gubernamental que tenga por objetivo mejorar la responsabilidad ante el público y facilitar el proceso de toma de decisiones por parte de los responsables de supervisar o iniciar acciones correctivas.

García, 2008

Uno de los aspectos que hace más atractiva una auditoría, lo Generar una propuesta que de constituye la posibilidad de generar una propuesta que de forma forma creativa responda a las creativa responda a las demandas del problema siempre condemandas del problema con criterio situacional, en el que se reconoce la influencia delcriterio situacional. entorno, expresado en diversos contextos (legislativo, político, económico, social, de la práctica u otros factores relevantes para el tema).

Elaboración propia a partir de la bibliografía consultada

De Armas (2008) señala cuatro objetivos que persigue la auditoría de gestión: 1. Evaluar la economía, la eficiencia y la eficacia de las entidades; 2. Evaluar el cumplimiento de las políticas gubernamentales; 3. Evaluar el cumplimiento de metas y resultados señalados en los programas, 
proyectos u operaciones de los organismos sujetos a control; 4. Analizar el costo de la actividad y el correcto uso dado a los recursos aprobados. (p.8)

Las organizaciones en general disponen de una planificación de actividades, en las que se establecen responsables y recursos a ser utilizados, de acuerdo al período de tiempo de duración de los proyectos plasmados en los planes operativos, tácticos y estratégicos, los mismos que necesitan ser evaluados con criterios técnicos e independientes, mediante la aplicación de indicadores de gestión que determinarán el grado de cumplimiento y por lo tanto medirán el cometido de la máxima autoridad; este tipo de auditoría es importante efectuarla y asumirla en las organizaciones en su conjunto o por áreas específicas, ya que dentro de las recomendaciones establecidas en el informe de auditoría se tratan los principios de eficiencia, efectividad, ética, economía y ecología con los que debe manejarse el ente económico. (Mayorga, Herrera, \& Álvarez, 2015, p.4)

De cara al contexto empresarial moderno, surge la corriente filosófica del enfoque social que permite el desarrollo integral de las organizaciones sin mirar su razón social o naturaleza, dentro de los autores contemporáneos que propone la inclusión del componente social dentro de la Auditoría de gestión se encuentra (Mayorga, 2018) quien presenta un modelo conceptual y procedimiento para la aplicación de la auditoría de gestión con enfoque de responsabilidad social donde se considera la evaluación objetiva e independiente que sustente el desarrollo de la visión estratégica de las organizaciones (Figura 1.) y (Figura 2.)

Figura 1. Modelo conceptual de Auditoría de Gestión.

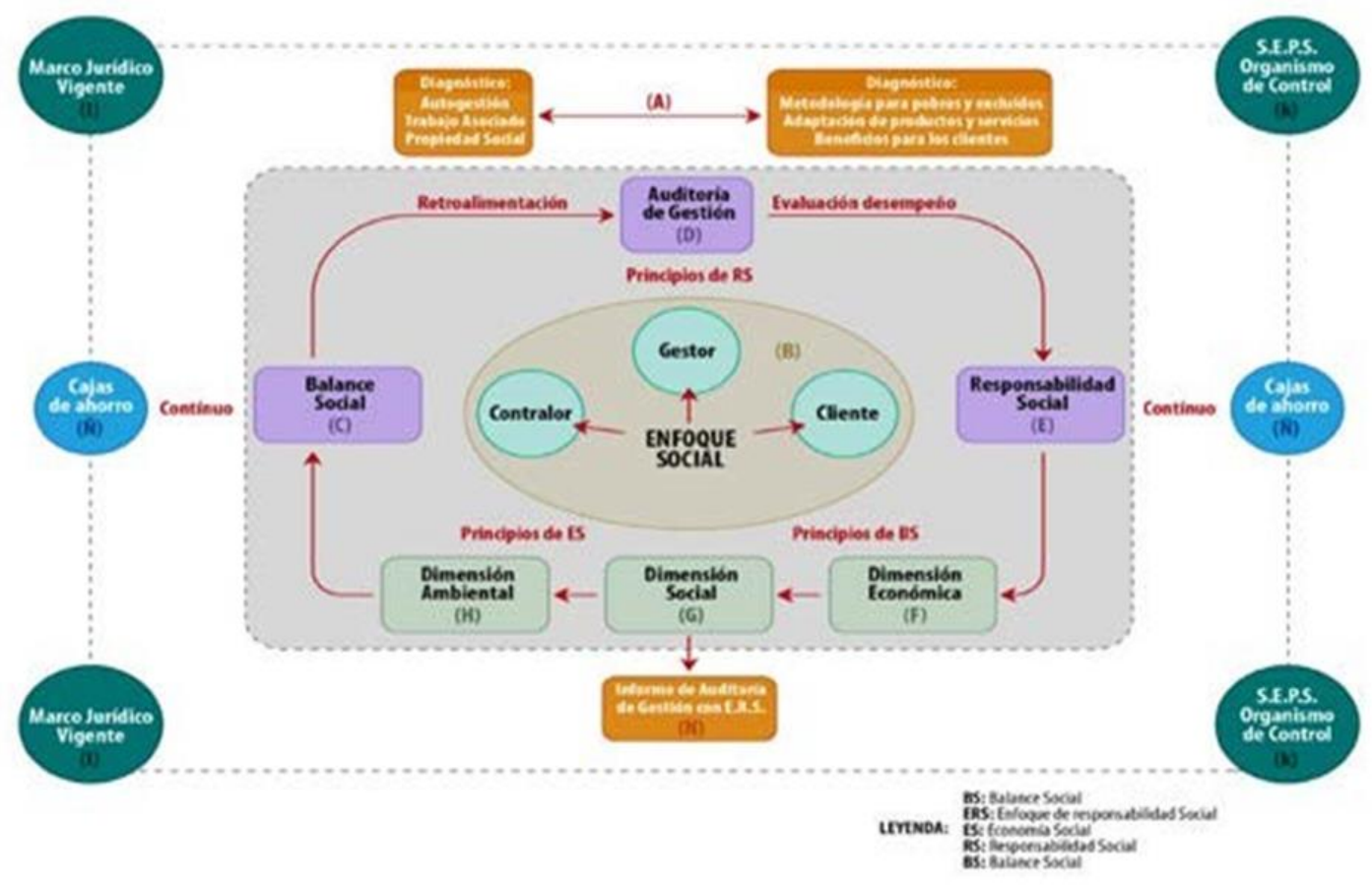

Fuente: Mayorga, 2018 
Figura 2. Procedimientos de Auditoría de Gestión.

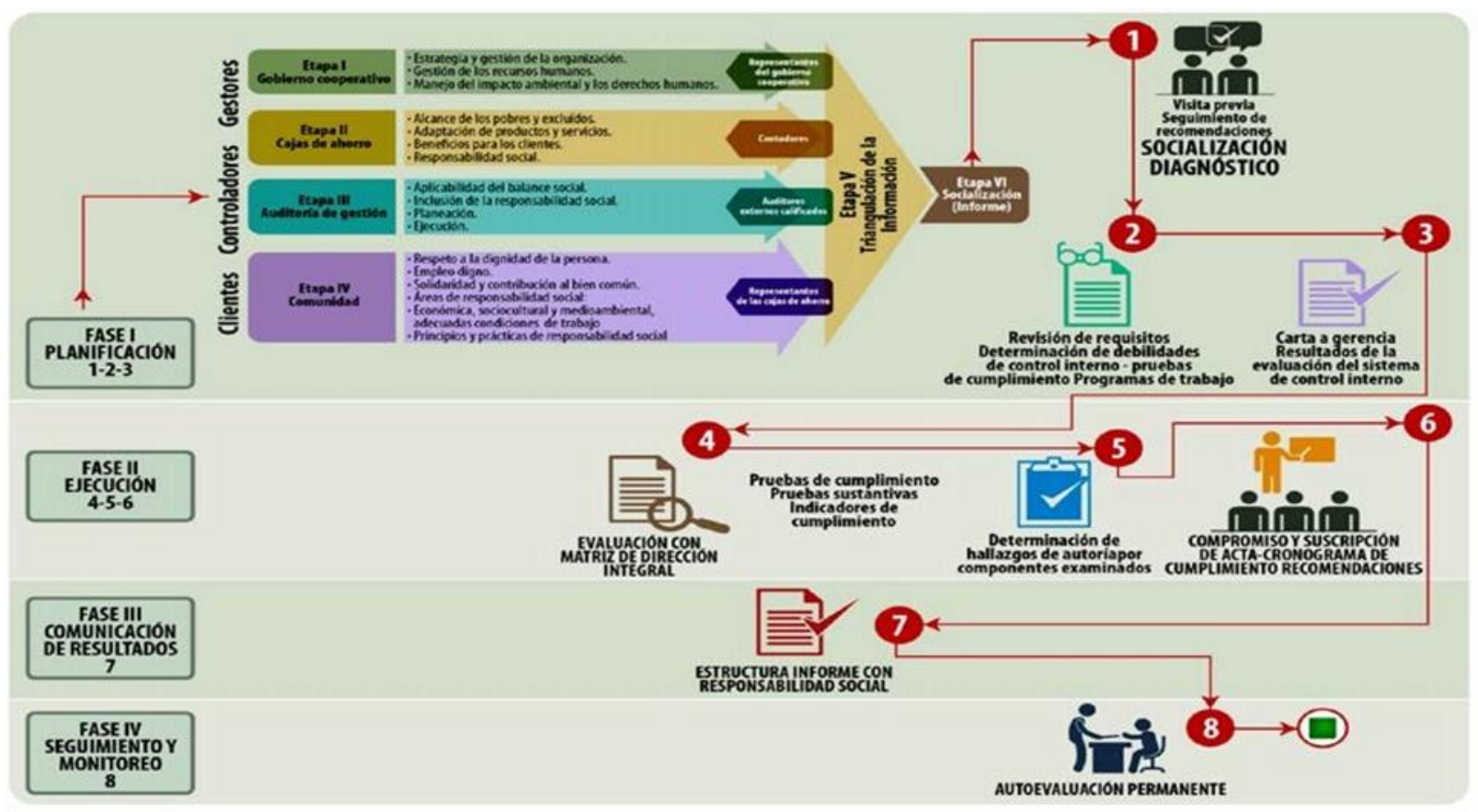

Fuente: Mayorga, 2018

\section{De cara a la relevancia de la temática, es necesario estudiar a autores clásicos del direccionamiento estratégico:}

Para resolver las deficiencias en la empresa se plantea un sistema de dirección que se conoce como Dirección Estratégica, cuyo objetivo principal es formular la estrategia y los planes de actuación empresarial, para prever acontecimientos futuros, desarrollándose la base en un plan estratégico explícito teniendo en cuenta los valores y la cultura organizacional vayan de la mano con dicho plan. (Laínez y Bellostas, 1991); (Goodstein, Nolan y Pfeiffer, 1998); (Aguilera \& Riascos, 2009)

En tal sentido la dirección estratégica es una disciplina multifuncional que se ocupa de la formulación, implantación y control de la estrategia, con el objeto de alcanzar una ventaja competitiva de carácter sostenible. Cuando se trabaja en las organizaciones bajo un enfoque estratégico, los temas centrales a considerar son: Dirección estratégica: Concepción del sistema de dirección que presupone un enfoque de orientación al entorno; Planificación estratégica: Utilización de procedimientos, métodos y tecnologías para llevar a cabo la dirección estratégica; Estrategia: Resultado del proceso y el medio para lograr el desarrollo de la organización. (Colectivo de autores, 2010,) (Mayorga, Herrera, \& Álvarez, 2015, p.1)

Un análisis de esta relación Planificación - Control a través de la auditoría, permite precisar, que, para las entidades del sistema financiero cooperativo, el proceso se retoma desde la última etapa del proceso del ciclo de dirección o proceso de decisión, que es el control. (Figura 3). 
Figura 3. La visión estratégica en la gestión de auditoría.

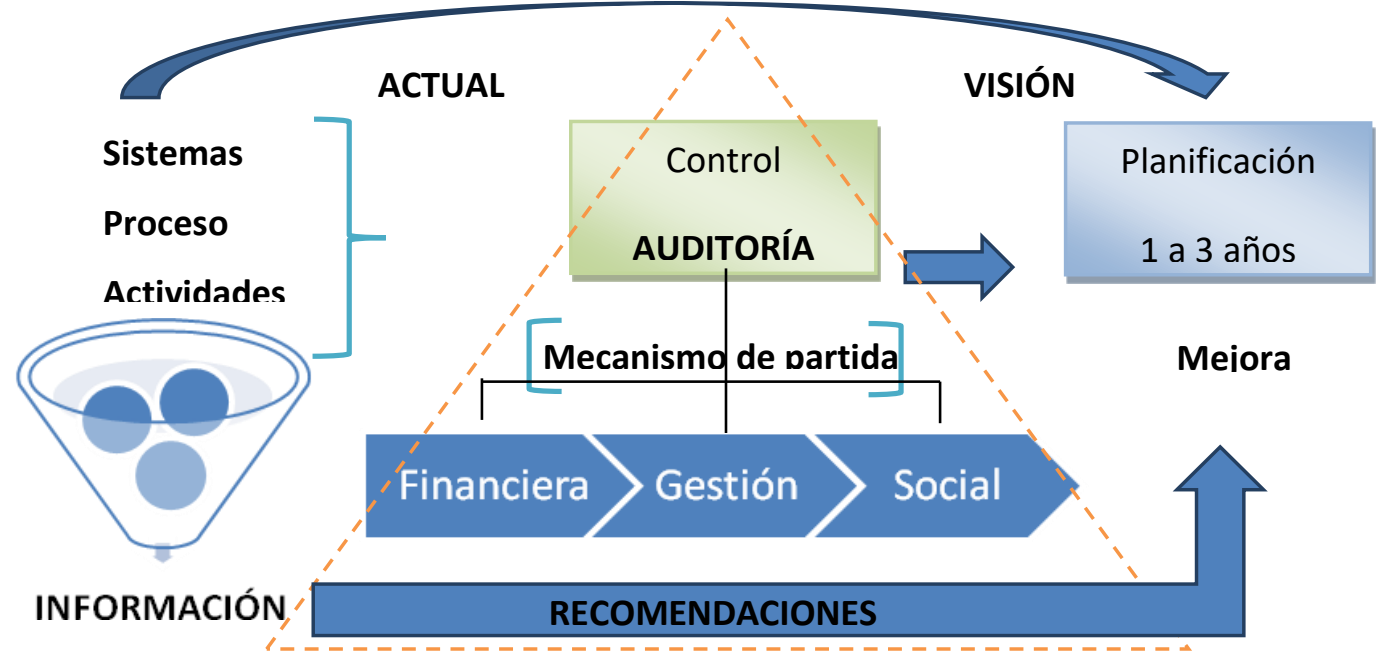

Fuente: Mayorga, Herrera, \& Ruso, (2016)

La gestión de la auditoría se relaciona también con la planificación, esto ocurre en el momento en que las recomendaciones o sugerencias de la auditoría forman parte de los insumos para la proyección de un nuevo periodo de ejecución y por tanto de nuevos patrones a auditar. "En tal sentido el plan de auditoría contendrá los objetivos (estrategia de la organización) y valorará el proceso de gestión, teniendo en cuenta la influencia del entorno, el uso de recursos y tiempo" (Mayorga, Herrera, \& Álvarez, 2015, p.8).

En el caso del director de la entidad, su misión consiste en controlar que los conocimientos, la experiencia y el know-how empleado en dar respuesta efectiva los problemas planteados (debilidades), lograr que se compartan, se socialice entre todos los especialistas y miembros de la organización involucrados, disponer de la recogida, enriquecimiento y almacenada de la memoria técnica que en el caso de la gestión de la auditoría será el informe su final. (Mayorga, Herrera, \& Álvarez, 2015, p.9)

Lo anterior está dado bajo la premisa de que los miembros de la organización están previamente involucrados, permitiendo al gestor (auditor interno) conformar el grupo "ad-hoc" para que respondan de forma rápida y oportuna ante las necesidades del decisor (alta dirección). No obstante, cabe la posibilidad de que uno de los problemas o la oportunidad que se presente, no encuentre respuesta en las áreas críticas determinadas y requiera asesoría externa (especialistas) para dar solución a lo detectado. (Mayorga, Herrera, \& Álvarez, 2015, p.10)

El énfasis en socializar y a la vez acumular la experiencia positiva para que sea reutilizada en procesos futuros, no ha sido preocupación de la alta dirección ni de los funcionarios hasta el momento. Actualmente se pretende dar soluciones emergentes, y en ocasiones sin detenerse para evaluar el costo-beneficio de la decisión tomada. (Mayorga, Herrera, \& Álvarez, 2015, p. 11) 
Existen otros elementos que apuntan al uso del enfoque estratégico en la gestión de las entidades del sistema financiero cooperativo, ello se explica desde las variables con la que interactúa la estrategia (Mayorga, Herrera, \& Álvarez, 2015), siendo estas:

Liderazgo: involucra a actores claves como el gestor (auditor) y decisor (alta dirección). Este soporte a nivel organizacional es el determinante para el éxito de una estrategia. Las destrezas, habilidades que se desarrollen, el carácter visionario y la capacidad movilizadora, podrán lograr que las recomendaciones o sugerencias que consten en la memoria del informe final de auditoría constituyan el insumo básico de un proceso decisorio (Mayorga, Herrera \& Álvarez, 2015, p.11).

Cultura: como elemento esencial para propiciar el intercambio y transferencia de información entre las personas, sobre la base de un compromiso real por cumplir las recomendaciones en el tiempo planteado. Esta cultura debe desarrollarse y reforzarse a partir del liderazgo que juegan los actores claves definidos anteriormente gestor y decisor. (Mayorga, Herrera \& Álvarez, 2015, p.11)

Sistema Político: considera el entorno, el contexto, marco jurídico regulador. Estos elementos se consideran como fuerzas que pueden dinamizar o complejizar el desarrollo de los procesos administrativos o financieros, por tanto, se debe velar por garantizar el buen funcionamiento del modelo. (Mayorga, Herrera \& Álvarez, 2015, p.11).

Sistema Administrativo: conjunto de elementos que garantizan, el funcionamiento básico de la gestión, como son: las personas (funcionarios, los socios, los directivos), la información, los procesos que intervienen (administrativos y/o financieros), las tecnologías, los problemas (debilidades y aspiraciones) y las oportunidades y fortalezas. (Mayorga, Herrera \& Álvarez, 2015, p.11).

Estructura: capacidad de adecuar funciones y actividades a los objetivos y estrategia de la entidad (Mayorga, Herrera \& Álvarez, 2015, p.11).

\section{El sector no financiero dinamiza la economía}

Con actividades enfocadas en la producción, transporte, prestación de servicios, construcción de viviendas y consumo, las organizaciones de la economía popular y solidaria (EPS) participan en el sistema económico del país. En la Superintendencia de Economía Popular y Solidaria están registradas, a junio de 2016, 8.325 organizaciones, entre asociaciones, cooperativas, organizaciones comunitarias y organismos de integración. Las organizaciones de la Economía Popular y Solidaria son parte del sector real de la economía del país, porque se dedican a la producción de bienes y servicios. Su particularidad es que están constituidas por personas que unieron recursos, conocimiento y fuerza de trabajo para realizar actividades económicas que les permita satisfacer sus necesidades en comunidad y mejorar sus condiciones de vida. Por su número, en primer lugar, están las organizaciones de producción, que representan el 58,5\% del total de organizaciones no financieras. Entre sus actividades principales destacan las textiles, agropecuarias, agrícolas, acuícolas y artesanales. En segundo lugar, con el 37,6\% del total, se 
encuentran las organizaciones dedicadas a brindar servicios, como transporte, limpieza, comercialización, mantenimiento, entre otros. (SEPS, 2018)

En consecuencia, es importante sintetizar el marco regulador vigente para el sector cooperativo de transporte. (tabla 2.)

Tabla 2. Normativa de las cooperativas.

\begin{tabular}{lll}
\hline Normativa & LegalPublicación & Extracto \\
Vigente &
\end{tabular}

Constitución de laRegistro oficial $\mathrm{N}^{\circ}$ Art. 309: "El sistema financiero nacional se compone de los sectores República del449 de fecha 20 depúblicos, privados, y del popular y solidario, que intermedian recursos Ecuador octubre del 2008. públicos" Art. 394.- "El estado garantizara la libertada de transporte terrestre, aéreo, marítimo y fluvial dentro del territorio nacional, sin privilegios de ninguna naturaleza. La promoción del transporte público masivo y la adopción de una política de tarifas diferenciadas de transporte serán prioritarias. El estado regulara el transporte terrestre, aéreo y acuático y las actividades aeroportuarias y portuarias"

Ley Orgánica deRegistro Oficial $\mathrm{N}^{\circ}$ Art.21.- Sector Cooperativo. - Es el conjunto de cooperativas entendidas Economía Popular y 444 del 10 de mayocomo sociedades de personas que se han unido en forma voluntaria para Solidaría del año 2011. satisfacer sus necesidades económicas, sociales y culturales en común, mediante una empresa de propiedad conjunta y de gestión democrática, con personalidad jurídica de derecho privado e interés social.

Reglamento a la Ley Registro oficial $\mathrm{N}^{\circ}$ Art. 32: "Estructura Interna. - Las cooperativas contarán con una Orgánica de648 del 27 de febrero Asamblea General de socios o de Representantes, un Consejo de Economía popular ydel año 2012 Administración, un Consejo de Vigilancia y una gerencia, cuyas solidaria atribuciones y deberes, además de las señaladas en esta Ley, constarán en su Reglamento y en el estatuto social de la cooperativa. En la designación de los miembros de estas instancias se cuidará de no incurrir en conflictos de intereses" Art. 33: "Asamblea General de Socios. - La Asamblea General es el máximo órgano de gobierno de la cooperativa y estará integrada por todos los socios, quienes tendrán derecho a un solo voto, sin considerar el monto de sus aportaciones y que podrá ser ejercido en forma directa o mediante delegación a otro socio. Sus decisiones y resoluciones obligan a todos los socios y a los órganos de la cooperativa" Art. 34."Asamblea General de representantes. - Las cooperativas que tengan más de doscientos socios, realizarán la asamblea general a través de representantes, elegidos en un número no menor de treinta, ni mayor de cien" Art. 38: "Consejo de Administración. - Es el órgano directivo y de fijación de políticas de la cooperativa, estará integrado por un mínimo de tres y máximo nueve vocales principales y sus respectivos suplentes, elegidos en Asamblea General en votación secreta, de acuerdo a lo establecido en el Reglamento de esta Ley"

\section{Elaboración propia a partir de la bibliografía consultada}




\section{Metodología.}

La lógica de la intervención del presente trabajo de investigación está conformada por un procedimiento de diagnóstico que evaluó los componentes del enfoque social dentro del desarrollo de las auditorías de gestión, en las unidades de análisis que en este caso corresponden las cooperativas de transporte de pasajeros; para el estudio de las aristas de investigación se determinaron como sigue:

1. Representantes del gobierno cooperativo (gerente, presidente, representante de los socios fundadores).

2. Personal del área financiera (contadores)

3. Auditores externos calificados por la SEPS

4. Representantes de la comunidad.

Se utilizó un procedimiento de diagnóstico para la medición de las variables que está estructurado en tres etapas: 1. en la primera etapa consta del Gobierno Cooperativo que es la estrategia y gestión de la organización, Gestión de los recursos humanos lo cual son los representantes del gobierno cooperativo, 2. en la etapa dos consta de Auditoría de Gestión con enfoque de responsabilidad social, Planeación Ejecución que son los Auditores externos calificados, 3. en la etapa tres consta de la triangulación de la información, en la etapa cuatro esta la socialización o informe. (figura 4.)

Figura 4. Procedimiento de diagnóstico del componente social

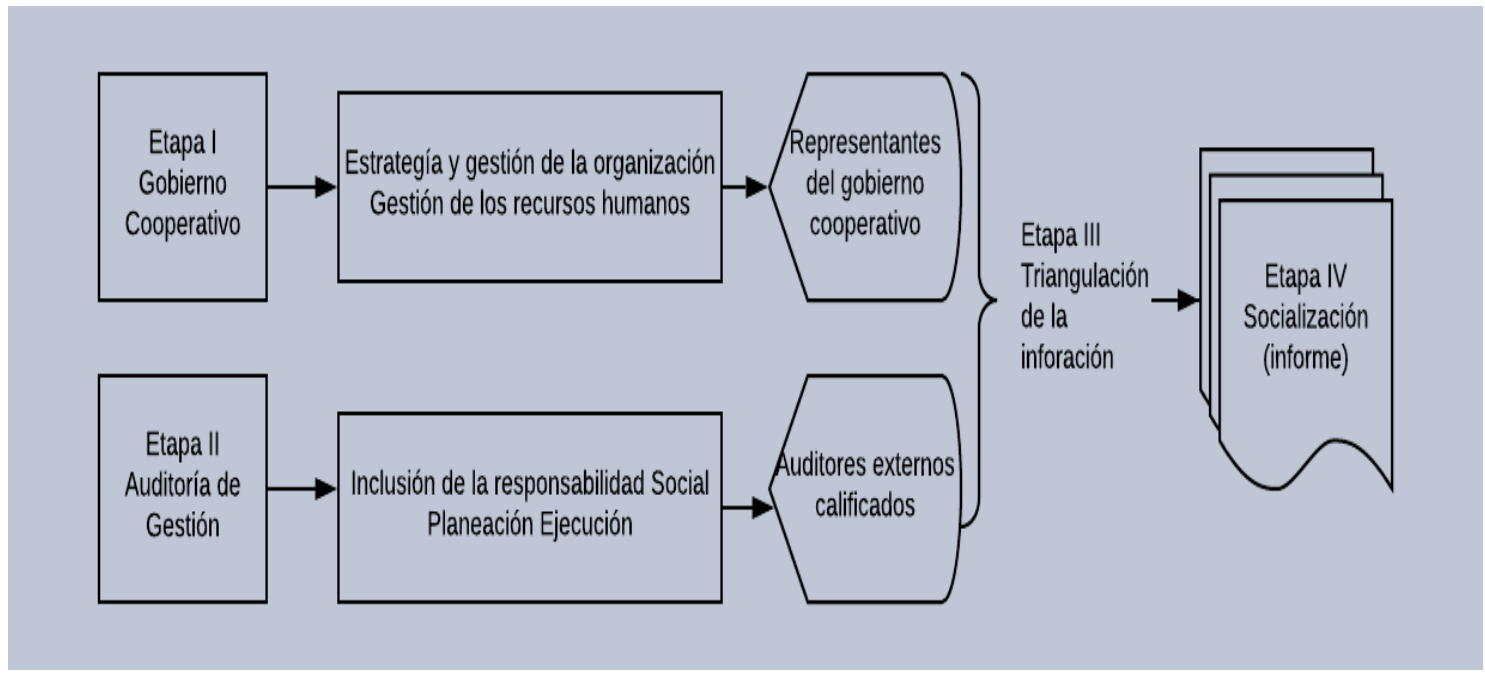

Elaboración propia

\section{Resultados.}

\section{Aplicación y análisis del diagnóstico}

Encuesta dirigida a al presidente, gerente, consejo de administración, consejo de vigilancia de la Cooperativa de Transportes Expreso Baños, quienes constituyen el gobierno cooperativo, se ha 
considerado necesario sintetizar los resultados de las encuestas en razón de las variables analizadas según el procedimiento de diagnóstico planteado.

1. Organización: conformado por el gerente, presidente, consejo de administración, consejo de vigilancia.

Figura 5. Organización

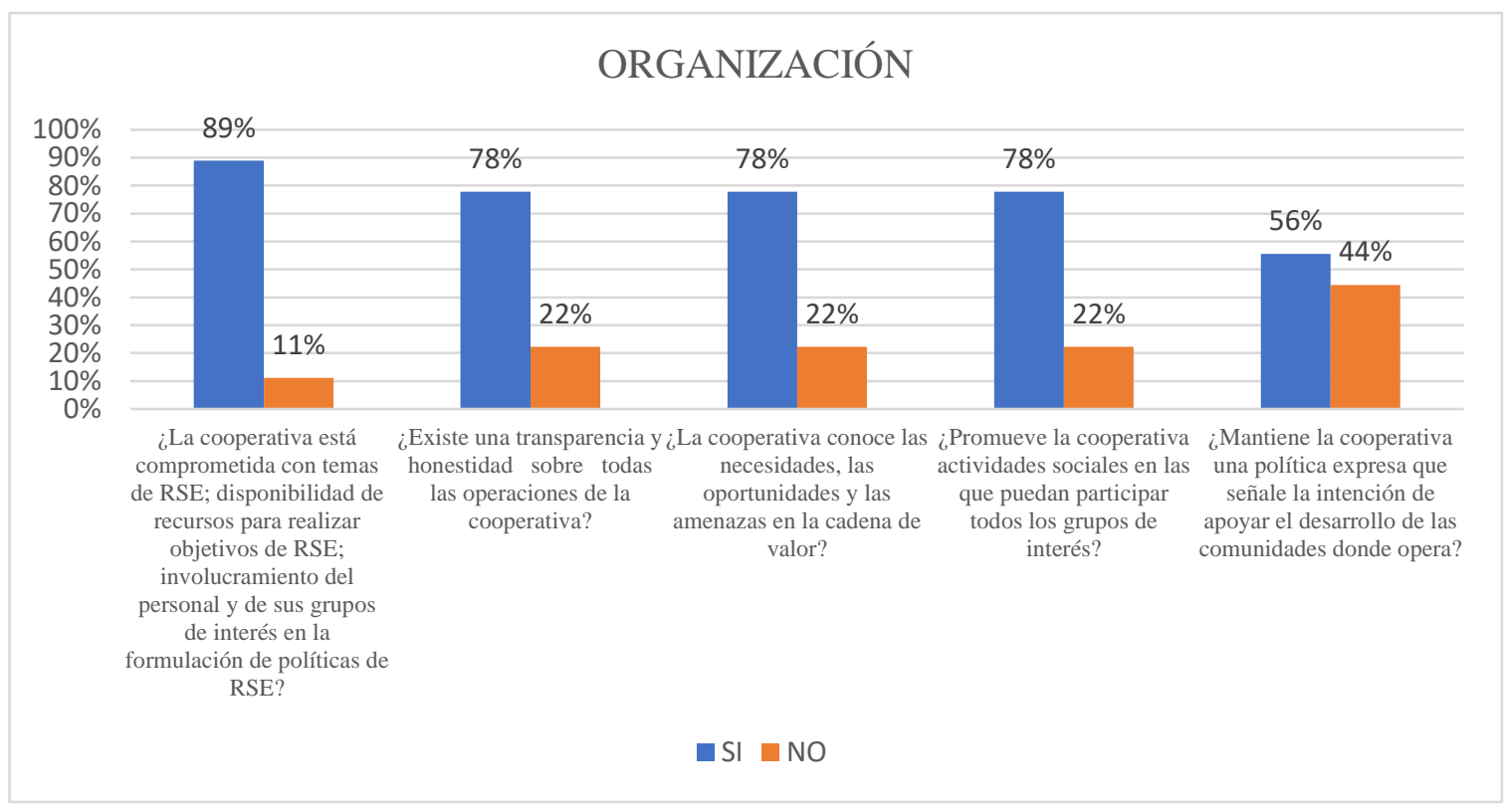

\section{Elaboración propia}

Lo que demuestra en la pregunta 1. que un $89 \%$ de los encuestados señalaron que la cooperativa está comprometida con temas del responsabilidad social, mientras que el $11 \%$ da a conocer que desconocen a cerca de los temas referentes a la disponibilidad del recurso, en la pregunta 2. el 78\% de los encuestados manifiestan que en la cooperativa existe transparencia y honestidad en sus operaciones, mientras que el $22 \%$ da a conocer que desconocen sobre la honestidad y transparencia en sus operaciones, pregunta 3. el $78 \%$ de los encuestados manifiestan que en la cooperativa conocen las necesidades y amenazas en la cadena de valor, mientras que el $22 \%$ da a conocer que desconocen sobre las necesidades y oportunidades en la cadena de valor, pregunta 4. el 78\% de los encuestados manifiestan que la cooperativa promueve actividades sociales, mientras que el $22 \%$ da a conocer que desconocen sobre la actividad social, pregunta 5. el 56\% de los encuestados manifiestan que la cooperativa mantiene una política para apoyar el desarrollo de las comunidades donde opera, mientras que el $44 \%$ da a conocer que desconocen sobre la política.

2. Estrategia y gestión de la organización: conformado por el gerente, presidente, consejo de administración, consejo de vigilancia. 
Figura 6. Estrategia y gestión de la organización

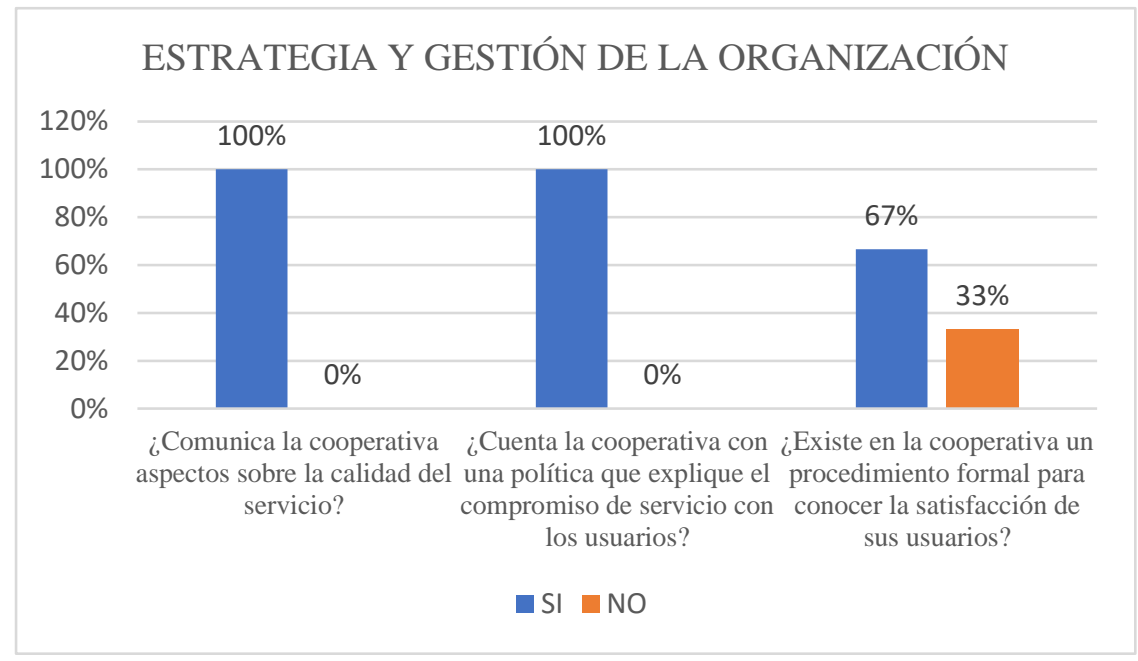

\section{Elaboración propia}

Una vez realizadas las encuestas se obtiene los siguientes resultados: en la pregunta 1; el 100\% de los encuestados manifiestan que la cooperativa comunica sobre la calidad del servicio, pregunta 2. el $100 \%$ de los encuestados manifiestan que la cooperativa cuenta con una política que explique el compromiso con los usuarios, pregunta 3. el 67\% de los encuestados manifiestan que la cooperativa conoce sobre el procedimiento formal para la satisfacción del usuario, mientras que el $33 \%$ da a conocer que desconocen sobre el procedimiento formal para dar a conocer la satisfacción de los usuarios.

3. Gestión de recursos humanos: conformado por el gerente, presidente Figura 7. Gestión de los recursos humanos

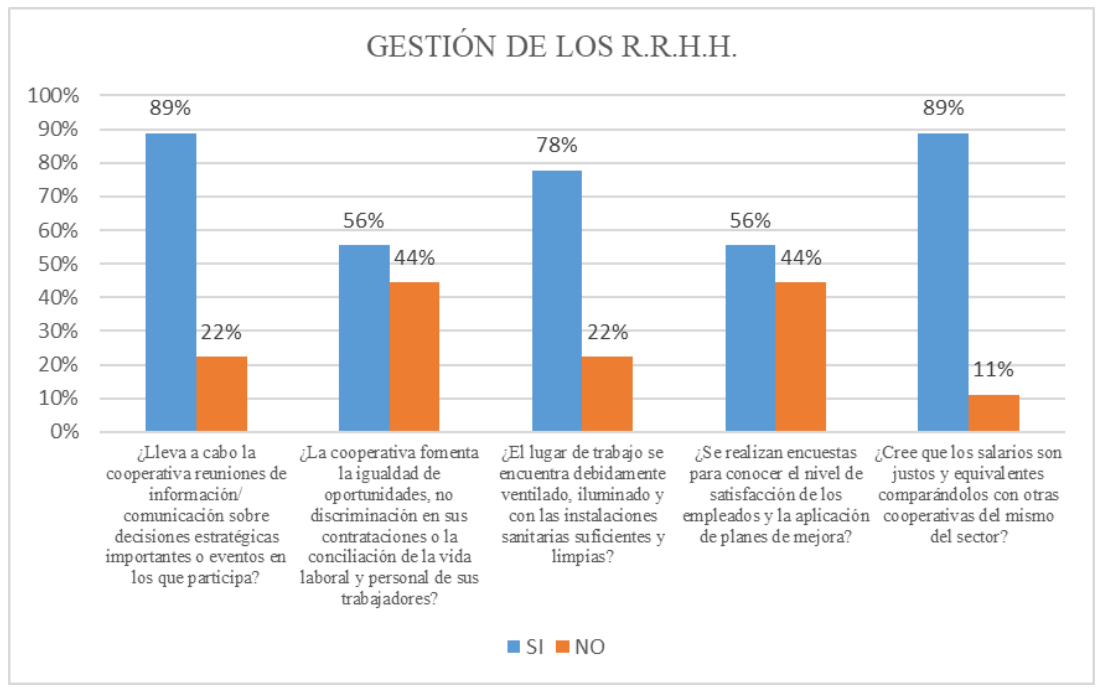

Elaboración propia 
En lo referente a la gestión de los recursos humanos se determinó: en la pregunta 1; el 80\% de los encuestados manifiestan que la cooperativa conoce sobre las reuniones de información para la toma de decisiones estratégicas, mientras que el $20 \%$ da a conocer que desconocen de las reuniones de información sobre las decisiones estratégicas, pregunta 2; el 56\% de los encuestados manifiestan que la cooperativa conoce sobre el procedimiento y las oportunidades en las contrataciones de personal, mientras que el $44 \%$ da a conocer que desconocen sobre la igual de oportunidades en las contrataciones, pregunta 3; el 78\% de los encuestados manifiestan que la cooperativa conoce sobre el lugar de trabajo y sus instalaciones que son adecuadas, mientras que el $22 \%$ da a conocer que desconocen sobre las instalaciones adecuadas y lugares de trabajo, pregunta 4; el 56\% de los encuestados manifiestan que la cooperativa conoce que se realizan encuestas para conocer el nivel de satisfacción de los empleados y la aplicación de planes de mejora, mientras que el $44 \%$ da a conocer que desconocen sobre la satisfacción de los empleados y la aplicación de planes de mejora, pregunta 5; el 89\% de los encuestados manifiestan que la cooperativa conoce sobre los sueldos justos y equivalentes comparado con otras cooperativas del mismo sector, mientras que el $33 \%$ da a conocer que desconocen sobre el procedimiento formal para dar a conocer la satisfacción de los usuarios.

A partir de los referentes teóricos y de la aplicación del procedimiento de diagnóstico de la inclusión del componente social en las cooperativas de transporte de pasajeros, corresponde contrastar los resultados con investigaciones efectuadas sobre el desempeño del personal en cooperativas de transporte en donde señalan que: " no cuenta con un manual de control interno, ni tampoco con un sistema que permita evaluar de una manera ágil y uniforme el desempeño de todos y cada uno de los prestadores de servicios de la institución, la mayoría de personas involucradas en la presente investigación están convencidas que la implantación de un sistema de evaluación del desempeño no solo influirá en la adecuada gestión del talento humano sino que también permitirá mejorar la gestión administrativa - financiera de la Cooperativa de Transportes y Turismo Baños en forma global " (Villacìs, 2011).

Por su parte en cuanto a las auditorías de gestión, se determina que no se sigue con un plan de asesoría, seguimiento y capacitación al departamento administrativo y socios, lo cual no permite un desempeño de calidad, en el proceso interno como en el servicio de transporte, se presenta el informe de planificación preliminar, especifica, ejecución de la auditoría y se emite el informe de auditoría con sus comentarios, conclusiones y recomendaciones, así mismo la evaluación del sistema de control interno permite determinar las áreas críticas de la cooperativa y emitir recomendaciones que permitan mejor el control, la aplicación de los indicadores de gestión a las diferentes áreas permite medir los niveles de eficiencia, eficacia y calidad que cuenta la cooperativa. (Peñafiel, 2017); (Muepàz, 2017)

La ejecución del marco jurídico entorno a las cooperativas de transporte de pasajeros, conlleva a la nueva institucionalidad comprometida a dar soporte al sector de la economía popular y solidaria, 
a través de la supervisión y control que se encuentra a cargo de la Superintendencia de Economía Popular y Solidaria, en donde se reorienta el control hacia los principios de Responsabilidad Social y cumplimiento de los objetivos estratégicos del Plan Nacional para el Buen Vivir 2013 - 2017. Las cooperativas de transporte de pasajeros consideradas unidades de análisis de esta investigación, son organizaciones autónomas que desarrollan actividades características de servicio de transporte para conectar a varios sectores a nivel nacional, sus directivos son miembros de la comunidad y sus operaciones son efectuadas con sus propias aportaciones.

En consecuencia del estudio efectuado, es prudente la aplicación de modelos de auditoría de gestión con enfoque social dentro de las actividades inherentes al direccionamiento estratégico, por encontrarse sustentadas en el marco regulador vigente del Ecuador; los elementos identificados anteriormente a pesar de estar reflejados en el contexto jurídico, no han sido diseñados o implantados con procedimientos específicos para las organizaciones de transporte de pasajeros, lo que ocasiona que estos entes no incorporen dentro de su gestión componentes de la realidad empresarial, tales como manejo del medio ambiente, recursos humanos, indicadores sociales, transparentación de la información hacia la comunidad, entre otros.

\section{Conclusiones.}

- La interrelación de los resultados de los instrumentos de diagnóstico permite detectar que: el marco regulador vigente permite la inclusión del enfoque social en la gestión de estas organizaciones, sin embargo están carentes políticas específicas por parte de los organismos de control, lo que se expresa en las insuficiencias y limitaciones que lastran la lógica integradora y sistémica de la auditoría de gestión con la responsabilidad social dada la escasa integración de un enfoque integrado en la evaluación del desempeño para las cooperativas de transporte de cara a la repercusión social de su gestión.

- De cara a la realidad empresarial como se ha señalado en el presente estudio, es menester que las memorias finales de la ejecución de la auditoría de gestión se conviertan en el soporte del direccionamiento estratégico en estas organizaciones y formarán parte de la visión estratégica de estas organizaciones.

- La carente inclusión del enfoque social en esta área pone de manifiesto la necesidad de aplicar el diagnóstico que se plantea en esta investigación, así como también el procedimiento de auditoría de gestión que incluye la medición del enfoque social generando un informe de cumplimiento que reúne el análisis de las dimensiones: social, económica y ambiental, en las organizaciones de transporte de pasajeros.

\section{Referencias bibliográficas.}

Aguilera, A., \& Riascos, S. (2009). Direccionamiento estratégico apoyado en las TIC. Revista de Estudios Gerenciales Scielo, 25(111), 1-10.

Arens, A., Elder, R., \& Beasley, M. (2007). Auditoría: Un enfoque integral. México: Pearson Educación.

Beltrán , J. (2005). Indicadores de Gestión . 3R . 
Contraloría General del Estado. (2015 de agosto de 2015). Obtenido de Manual de Auditoría de Gestión

http://www.contraloria.gob.ec/documentos/normatividad/manual\%20de\%20auditoria\%20 de\%20gestion.pdf

De Armas, R. (2008). Auditoría de Gestión, conceptos y métodos. Habana: Félix Varela.

Departamento Administrativo Nacional de Estadística DANE. (enero de 2014). Metodología para el seguimiento y evaluación de políticas, planes y proyectos públicos. Departamento Administrativo Nacional de Estadística DANE. Bógota, Colombia: Oficina de las Naciones Unidades contra la Droga y el Delito.

Estupiñan, R. (2006). Control Interno y fraudes con base en los ciclos transaccionales. México: Ecoe Ediciones.

Fonseca, O. (2007). Auditoria Gubernamental Moderna. Lima: Instituto de Investigación en Accountability y Control - IICO.

Goodstein, L., Nolan, T., \& Pfeiffer, W. (1998). Planeación estratégica aplicada. México: McGraw-Hill.

Laínez G., J., \& Bellostas P., A. (1991). La planificación y gestión estrategica en grupos multinacionales: Los modelos de simulación. Revista Española de Financiación y Contabilidad, 501-534.

Maldonado, M. (2011). Auditoría de Gestión (3 ed.). Quito: Luz de América.

Mayorga, M., Álvarez, G., Mayorga, L., \& Herrera , K. (2018). Auditoría de gestión con un enfoque de responsabilidad social. Quito: Editorial Jurídica del Ecuador.

Mayorga, M., Álvarez, G., Saltos , J., \& Chiliquinga, E. (2015). Perspectivas de la Auditoria de Gestión con un enfoque de responsabilidad social en el sistema financiero cooperativo ecuatoriano. Ambato: Repositorio Universidad Regional Autónom de los Andes, UNIANDES.

Mayorga, M., Herrera, K., \& Álvarez, G. (2015). La auditoria como soporte estratégico en la gestión del sistema financiero cooperativo. III Congreso Científico Internacional Uniandes (págs. 1-15). Ambato: Repositorio Universidad Autónoma de los Andes.

Mayorga, M., Herrera, K., \& Ruso, F. (2016). La auditoría como soporte estratégico en la gestión del sistema financiero cooperativo de la provincia de Tungurahua. Revista Cubana de Contabilidad y Finanzas, 10(1), 15-27.

Sandoval, H. (2012). Introducción a la auditoría. México: Red Tercer Milenio.

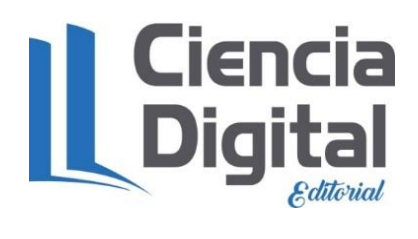


Vol. 4, $\mathrm{N}^{\circ}$ 2, p. 40-56, abril - junio, 2020

PARA CITAR EL ARTÍCULO INDEXADO.

Mayorga Díaz, M. P., Hervas Paredes, E. A., López Paredes, M. A., \& Toaza Tipantasig, S. E. (2020). Interacción de la auditoría de gestión en el direccionamiento estratégico de cooperativas de transporte. Visionario Digital, 4(2), 40-56. https://doi.org/10.33262/visionariodigital.v4i2.1214

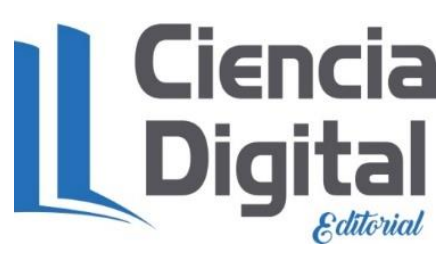

El artículo que se publica es de exclusiva responsabilidad de los autores y no necesariamente reflejan el pensamiento de la Revista Visionario Digital.

El artículo queda en propiedad de la revista y, por tanto, su publicación parcial y/o total en otro medio tiene que ser autorizado por el director de la Revista Visionario Digital.
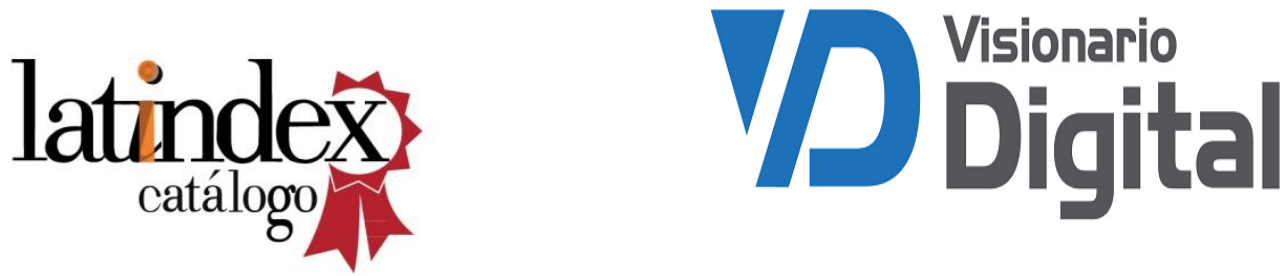